\title{
An unconditional proof of the Andre-Oort conjecture for Hilbert modular surfaces
}

Article

Accepted Version

Daw, C. and Yafaev, A. (2011) An unconditional proof of the Andre-Oort conjecture for Hilbert modular surfaces.

Manuscripta Mathematica, 135 (1). pp. 263-271. ISSN 00252611 doi: https://doi.org/10.1007/s00229-011-0445-x Available at https://centaur.reading.ac.uk/70355/

It is advisable to refer to the publisher's version if you intend to cite from the work. See Guidance on citing.

Published version at: http://link.springer.com/article/10.1007/s00229-011-0445-x

To link to this article DOI: http://dx.doi.org/10.1007/s00229-011-0445-x

Publisher: Springer-Verlag

All outputs in CentAUR are protected by Intellectual Property Rights law, including copyright law. Copyright and IPR is retained by the creators or other copyright holders. Terms and conditions for use of this material are defined in the End User Agreement.

\section{www.reading.ac.uk/centaur}

\section{CentAUR}

Central Archive at the University of Reading

Reading's research outputs online 


\title{
An unconditional proof of the André-Oort conjecture for Hilbert modular surfaces.
}

\author{
Christopher Daw and Andrei Yafaev*
}

March 12, 2016

\section{Introduction.}

The purpose of this paper is to prove the following special case of the AndréOort conjecture

Theorem 1.1 Let $S$ be a Hilbert modular surface and $C \subset S$ an irreducible algebraic curve containing an infinite set of special points. Then $C$ is a special subvariety of $S$.

We refer to [3] for the notions of special points and special subvarieties. For background on on Hilbert modular surfaces we refer to [3] and [16]. For generalities, history and results obtained so far on the André-Oort conjecture, we refer to [14], [15] and [8].

Theorem 1.1 was proved by Edixhoven in [3] under the assumption of the Generalised Riemann Hypothesis. Recently Klingler, Ullmo and the second author proved the André-Oort conjecture in full generality under the assumption of the GRH (see [13] and [6]). In this paper we give an unconditional proof of the André-Oort conjecture for Hilbert modular surfaces using the ideas of Pila (see [10] and [11]) and results of Peterzil-Starchenko, Pila-Wilkie, Edixhoven, Ullmo and the second author.

Let us briefly outline the strategy. Let $F$ be a real quadratic field, $O_{F}$ its ring of integers and $\Gamma:=\mathrm{SL}_{2}\left(O_{F}\right)$. By Hilbert modular surface we

\footnotetext{
*University College London, Department of Mathematics, Gower street, WC1E 6BT London, United Kingdom, e-mails: c.daw@ucl.ac.uk, yafaev@ucl.ac.uk
} 
mean $S=\Gamma \backslash \mathbb{H}^{2}$. This is a connected component of the Shimura variety $S h_{K}(G, X)$ defined by the Shimura datum $(G, X)=\left(\operatorname{Res}_{F / \mathbb{Q}} \mathrm{GL}_{2, F}, \mathbb{H}^{ \pm 2}\right)$ and $K=\mathrm{GL}_{2}\left(\widehat{O_{F}}\right)$. One can also consider quotients of $\mathbb{H} \times \mathbb{H}$ by other congruence subgroups of $\mathrm{SL}_{2}\left(O_{F}\right)$. The André-Oort conjecture for such quotients is equivalent to the one for $S$ (see Proposition 2.1 of [4]). Furthermore, as a subvariety is special if and only if irreducible components of its images by Hecke correspondences are special, the statement holds for a curve contained in any component of $S h_{K}(G, X)$.

Let us outline our strategy. The Shimura variety $S h_{K}(G, X)$ is a coarse moduli space for pairs $(A, i)$ where $A$ is an abelian surface and $i: O_{F} \longrightarrow$ $\operatorname{End}(A)$ is a morphism. It admits a canonical model over $\mathbb{Q}$ and $S$ is defined over a certain explicit abelian extension. Let $\pi: \mathbb{H}^{2} \longrightarrow S$ be the uniformisation map. We choose a certain fundamental set $\mathcal{F} \subset \mathbb{H}^{2}$ (actually a certain part thereof) for the action of $\Gamma$. Let $C$ be a curve in $S$ containing an infinite set $\Sigma$ of special points (in particular $C$ is defined over a number field). We let $\mathcal{Z}:=\pi^{-1} C \cap \mathcal{F}$ and $\mathcal{Z}^{\text {alg }}$ the algebraic part of $\mathcal{Z}$ i.e. the union of all real semi-algebraic subsets contained in $\mathcal{Z}$ when $\mathbb{H} \times \mathbb{H}$ is viewed as a subset of $\mathbb{R}^{4}$. Suppose that $C$ is not special. A result of Ullmo and the second author then implies that $\mathcal{Z}^{\text {alg }}$ contains no special points (see section 3). A theorem of Peterzil and Starchenko shows that $\mathcal{Z}$ is definable in a certain o-minimal structure on $\mathbb{R}^{4}$ (see section 4 ). Then, by a theorem of Pila and Wilkie, the number of algebraic points on $\mathcal{Z}$ of degree at most four and up to a height $T$ is $\ll_{\epsilon} T^{\epsilon}$ for any $\epsilon>0$. For a special point $x$ of $S$, we let $\left(A_{x}, i_{x}\right)$ be the corresponding pair as above. The ring $\operatorname{End}_{O_{F}}\left(A_{x}\right)$ of endomorphisms commuting with the action of $O_{F}$ is an order in a totally imaginary quadratic extension of $F$. We let $d_{x}:=\left|\operatorname{Disc}\left(\operatorname{End}_{O_{F}}\left(A_{x}\right)\right)\right|$. In section 2 we show that the height of a special point in $\mathcal{F}$ is bounded by a power of its discriminant. Hence PilaWilkie's result shows that the size of the Galois orbit of the special point $x$ is $\ll_{\epsilon} d_{x}^{\epsilon}$ where $d_{x}$ is the discriminant of $x$ and $\epsilon$ can be chosen arbitrary small. This contradicts the result of Edixhoven who showed that the size of the Galois orbit is $\gg d_{x}^{1 / 8}$. It seems very likely that the methods of this paper generalise to the mixed case i.e. the analog of the André-Oort conjecture for the universal abelian scheme over a Hilbert modular surface. To generalise the result to the case of Hilbert modular varieties of higher dimension, one needs unconditional lower bounds for the Galois orbits of special points in terms of a positive power of the discriminant. Obtaining such bounds seems to be a very hard problem. 
We would like to express our thanks to Jonathan Pila for explaining his method to us and for interesting discussions on the subject of this paper. We are extremely grateful fo Emmanuel Ullmo for several very useful discussions and remarks.

\section{Bounds on the heights of special points.}

In this section we give upper bounds on the height of coordinates of special points contained in a certain fundamental set in terms of 'their discriminant'. For an element $\alpha$ of $F$, we denote by $\alpha^{\prime}$ the image of $\alpha$ by the non-trivial automorphism of $F$. A point $z=\left(z_{1}, z_{2}\right)$ of $\mathbb{H}^{2}$ is called special if $\pi(z)$ is a special point of $S$. Let $z=\left(z_{1}, z_{2}\right)$ be a special point in $\mathbb{H}^{2}$. Then $z$ is fixed by a certain semisimple element of $\mathrm{SL}_{2}(F)$. From this it immediately follows that $z_{1}$ satisfies an equation $a z_{1}^{2}+b z_{1}+c=0$ with $a, b, c \in O_{F}$ and $z_{2}$ satisfies $a^{\prime} z_{2}^{2}+b^{\prime} z_{2}+c^{\prime}=0$. The field $K=F\left(z_{1}\right)$ is an imaginary quadratic extension of $F$.

We follow [17], section 1.1. Let $z=\left(z_{1}, z_{2}\right)$ be a point of $\mathbb{H}^{2}$ and consider the embedding $\mathcal{L}_{z}: F \times F \longrightarrow \mathbb{C}^{2}$ sending $(\alpha, \beta)$ to $\left(\alpha z_{1}+\beta, \alpha^{\prime} z_{2}+\beta^{\prime}\right)$. To a point $z=\left(z_{1}, z_{2}\right)$, on associates the complex torus

$$
A_{z}=\mathbb{C}^{2} / \mathcal{L}_{z}\left(O_{F} \oplus \mathcal{I}\right)
$$

where $\mathcal{I}$ is an invertible rank one $O_{F}$-module contained in $O_{F}^{\vee}$, the $\mathbb{Z}$-dual of $O_{F}$ with respect to the trace. The action of $O_{F}$ on $A_{z}$ is given by $m(a):\left(\zeta_{1}, \zeta_{2}\right) \longrightarrow\left(a \zeta_{1}, a^{\prime} \zeta_{2}\right)$. In [17], section 1.1 , it is shown that $A_{z}$ is a polarised abelian variety. By [16], section I.7, corollary 7.3, the abelian variety corresponding to a point $x$ of the component $S$ of $S h_{K}(G, X)$ is $A_{z}=\mathbb{C}^{2} / \mathcal{L}_{z}\left(O_{F} \oplus O_{F}\right)$ where $z \in \pi^{-1}(x)$.

Suppose that $z$ is a special point. Denoting $\Lambda_{z}:=\mathcal{L}_{z}\left(O_{F} \oplus O_{F}\right)$, we have

$$
\operatorname{End}_{O_{F}}\left(A_{z}\right)=\left\{k \in K: k \Lambda_{z} \subset \Lambda_{z}\right\}
$$

The ring $\operatorname{End}_{O_{F}}\left(A_{z}\right)$ is an order in $K$ containing $O_{F}$. We first prove the following:

Lemma 2.1 The relative discriminant ideal $\operatorname{Disc}_{K / F}\left(\operatorname{End}_{O_{F}}\left(A_{z}\right)\right) O_{F}$ is generated by the $b^{2}-4 a c$ where $a z_{1}^{2}+b z_{1}+c=0$ (with $a, b, c \in O_{F}$ ) is a quadratic equation satisfied by $z_{1}$. 
Proof. Let $R$ be $\operatorname{End}_{O_{F}}\left(A_{z}\right)$ and let $I$ be the ideal in $O_{F}$ generated by the $b^{2}-4 a c$ where $a z_{1}^{2}+b z_{1}+c=0$ (with $a, b, c \in O_{F}$ ) be any equation satisfied by $z_{1}$. For any such equation, $R$ contains $a z_{1}$ and hence the relative discriminant ideal $\operatorname{Disc}_{K / F}(R) O_{F}$ contains $I$.

To prove the other inclusion, fix a prime ideal $P$ of $O_{F}$ and let $O_{F, P}$ be the completion of $O_{F}$ at $P$. We let $M$ be a maximal ideal of $O_{K}$ above $P$ and $K_{M}$ the completion of $K$ with respect to the corresponding valuation. Let $a z_{1}^{2}+b z_{1}+c=0$ be an equation satisfied by $z_{1}$ with $v_{P}(a b c)$ minimal $\left(v_{P}\right.$ denotes the $P$-adic valuation). It follows, in particular, that $a, b$ and $c$ are relatively prime in the ring $O_{F, P}$. Then, the proof of lemma 7.5 of [2] goes through and shows that the local order $\left\{k \in K_{M}: k\left(\Lambda_{z} \otimes O_{F, P}\right) \subset \Lambda_{z} \otimes O_{F, P}\right\}$ is $O_{F, P}\left[a z_{1}\right]$. It follows that $\operatorname{Disc}_{K / F}(R) O_{F, P}$ is generated by $b^{2}-4 a c$, hence is contained in $I O_{F, P}$. As this holds for all primes $P$, we conclude that $\operatorname{Disc}_{K / F}(R) O_{F}=I$

We write $z_{i}=x_{i}+i y_{i}$ and we define

$$
H(z):=\max \left(H\left(x_{1}\right), H\left(x_{2}\right), H\left(y_{1}\right), H\left(y_{2}\right)\right)
$$

where $H$ denotes the standard multiplicative height of an algebraic number (see [1], Chapter I). Our aim is to give an upper bound for $H(z)$ for $z$ in a fundamental set for $\Gamma$ in terms of a power of $d_{z}:=\left|\operatorname{Disc}\left(\operatorname{End}_{O_{F}}\left(A_{z}\right)\right)\right|$.

Choose an equation $a z_{1}^{2}+b z_{1}+c=0$ where $a, b, c$ are such that the norm $\left|N_{F / \mathbb{Q}}\left(b^{2}-4 a c\right)\right|$ is minimal. The above discussion shows that

$$
\left|N_{F / \mathbb{Q}}\left(\operatorname{Disc}_{K / F}\left(\operatorname{End}_{O_{F}}\left(A_{z}\right)\right)\right)\right|=\left|N_{F / \mathbb{Q}}\left(b^{2}-4 a c\right)\right|
$$

In [5], Chapter I, Proposition 2.11, it is proved that there exists a fundamental set (that is, a set containing a fundamental domain) for the action of $\Gamma=\mathrm{SL}_{2}\left(O_{F}\right)$ of the form

$$
\mathcal{K} \cup V_{1} \cup \ldots \cup V_{h}
$$

where $h$ is the class number of $F, \mathcal{K}$ is compact and the $V_{i}$ are the so-called cusp sectors.

Here $V_{1}$ is the cusp sector at infinity $\infty$. By definition, there is a constant $C>0$ and $T>0$ such that

$$
V_{1}=\left\{\left(z_{1}, z_{2}\right) \in \mathbb{H} \times \mathbb{H}: y_{1} y_{2}>C,\left|x_{1}\right| \leq T,\left|x_{2}\right| \leq T\right\}
$$


Noticing that on $\mathcal{K}, y_{i}$ s are bounded below and the $\left|x_{i}\right| \mathrm{s}$ are bounded, we may and do (after possibly altering $C$ and $T$ ), assume that $\mathcal{K} \subset V_{1}$. Furthermore, for $\epsilon \in O_{F}^{*}$, the transformation $\left(z_{1}, z_{2}\right) \mapsto\left(\epsilon^{2} z_{1}, \epsilon^{-2} z_{2}\right)$ is in $\Gamma$. We can therefore assume that $\left(y_{1}, y_{2}\right)$ is in the fundamental set for the action $\left(y_{1}, y_{2}\right) \mapsto\left(\epsilon^{2} y_{1}, \epsilon^{-2} y_{2}\right), \epsilon \in O_{F}^{*}$. We therefore have an inequality

$$
A^{-1} \leq \frac{y_{i}^{2}}{y_{1} y_{2}} \leq A
$$

where $A$ is a constant depending on $F$ only. For reasons explained in section five, for our purposes it is enough to consider the special points in $\mathcal{K} \cup V_{1}$. According to the discussion above, we consider special points in the set

$$
\mathcal{F}=\left\{\left(z_{1}, z_{2}\right) \in \mathbb{H}^{2}: y_{1} y_{2}>C, A^{-1} \leq \frac{y_{i}^{2}}{y_{1} y_{2}} \leq A,\left|x_{1}\right| \leq T,\left|x_{2}\right| \leq T\right\}
$$

Theorem 2.2 There exists a real $c_{1}>0$ such that for any special point $z=\left(z_{1}, z_{2}\right) \in \mathcal{F}$ we have,

$$
H(z) \leq c_{1} d_{z}^{1 / 4}
$$

Remark 2.3 The proof below generalises to the case of Hilbert modular varieties of arbitrary dimension.

Proof. On $\mathcal{F}$, we have

$$
y_{1}^{2}, y_{2}^{2} \geq U=A^{-1} C
$$

Let $a z_{1}^{2}+b z_{1}+c=0$ with $a, b, c \in O_{F}$ be the equation satisfied by $z_{1}$ with $\left|N_{F / \mathbb{Q}}\left(b^{2}-4 a c\right)\right|$ minimal. Then $z_{2}$ satisfies $a^{\prime} z_{2}^{2}+b^{\prime} z_{2}+c^{\prime}=0$ and we let $D_{1}=$ $\left|b^{2}-4 a c\right|$ and $D_{2}=\left|b^{\prime 2}-4 a^{\prime} c^{\prime}\right|$. We have $\left|N_{F / \mathbb{Q}}\left(\operatorname{Disc}_{K / F}\left(\operatorname{End}_{O_{F}}\left(A_{z}\right)\right)\right)\right|=$ $D_{1} D_{2}$ and

$$
d_{z}=D_{1} D_{2} \Delta_{F}^{2}
$$

where $\Delta_{F}=\left|\operatorname{Disc}\left(O_{F}\right)\right|$.

Note that we have

$$
|b|=2|a|\left|x_{1}\right| \leq 2 T|a|,\left|b^{\prime}\right|=2\left|a^{\prime}\right|\left|x_{2}\right| \leq 2 T\left|a^{\prime}\right|
$$

Secondly, since $D_{1}=4 a^{2} y_{1}^{2}$ and $D_{2}=4 a^{\prime 2} y_{2}^{2}$, we have

$$
|a| \leq \sqrt{\frac{D_{1}}{4 U}},\left|a^{\prime}\right| \leq \sqrt{\frac{D_{2}}{4 U}}
$$


We have

$$
H\left(x_{1}\right)^{2} \leq\left|4 a a^{\prime} \frac{b b^{\prime}}{4 a a^{\prime}}\right|=\left|b b^{\prime}\right| \leq 4 T^{2}\left|a a^{\prime}\right| \leq T^{2} \frac{\sqrt{D_{1} D_{2}}}{U}
$$

hence

$$
H\left(x_{1}\right) \leq \frac{T}{\sqrt{U}}\left(D_{1} D_{2}\right)^{1 / 4}=\frac{T}{\sqrt{U \Delta_{F}}} d_{z}^{1 / 4}
$$

Finally,

$$
H\left(y_{1}\right)^{4} \leq 16 a^{2} a^{\prime 2} \leq \frac{D_{1} D_{2}}{U^{2}}
$$

Hence

$$
H\left(y_{1}\right) \leq \frac{1}{\sqrt{U \Delta_{F}}} d_{z}^{1 / 4}
$$

The argument proceeds identically for $x_{2}$ and $y_{2}$. For $c_{1}$ it suffices to take $\max \left(\frac{T}{\sqrt{U \Delta_{F}}}, \frac{1}{\sqrt{U \Delta_{F}}}\right)$.

\section{Characterisation of special subvarieties.}

In this section we show that proper special subvarieties of $S$ may be characterised by the property that the 'algebraic part' of their preimages in $\mathbb{H}$ contains no special points.

Let $C$ be an irreducible algebraic curve in $S$ and let $\mathcal{Z}:=\pi^{-1} C$. Let $\left(\tau_{1}, \tau_{2}\right)$ be a point of $\mathbb{H}^{2}$. Writing $\tau_{1}=x+i y$ and $\tau_{2}=u+i v$, we can view $\mathbb{H}^{2}$ as a subset of $\mathbb{R}^{4}$. A semi-algebraic subsets of $\mathbb{H}^{2} \subset \mathbb{R}^{4}$ is by definition the intersection of a semi-algebraic subsets of $\mathbb{R}^{4}$ with $\mathbb{H}^{2}$. Following Pila, we define $\mathcal{Z}^{\text {alg }}$ to be the union of all connected positive dimensional semi-algebraic subsets of $\mathcal{Z}$. We also define $\mathcal{Z}^{c a}$ to be the union of positive dimensional complex algebraic subsets contained in $\mathcal{Z}$. The argument of the proof of Proposition 2.2 of [10] shows that

$$
\mathcal{Z}^{a l g}=\mathcal{Z}^{\text {ca }}
$$

The characterisation we are going to use is the following.

Theorem 3.1 If $C$ is not special then $\mathcal{Z}^{\text {alg }}$ contains no special points.

Proof. As remarked above $\mathcal{Z}^{c a}=\mathcal{Z}^{\text {alg }}$. Suppose that $\mathcal{Z}^{c a}$ is not empty (otherwise there is nothing to prove). Let $\mathcal{Z}^{\prime}$ be an analytic component of 
$\mathcal{Z}^{c a}$. As the dimension of $\mathcal{Z}$ is one, $\pi\left(\mathcal{Z}^{\prime}\right)=\pi(\mathcal{Z})=C$. In particular $\pi\left(\mathcal{Z}^{\prime}\right)$ is an algebraic subvariety of $S$. By [12], $\pi\left(\mathcal{Z}^{\prime}\right)=C$ is a weakly special subvariety of $S$. By [7], theorem 4.3, a weakly special subvariety (or totally geodesic in Moonen's terminology) of a Shimura variety is special (or Hodge type) if and only if it contains a special point. Therefore, if $C$ is not special, then $C$, and hence $\mathcal{Z}^{\text {alg }}$, contains no special points.

\section{Definability.}

We refer to section 3 of [10] and references contained therein for notions of o-minimal structures and definability. We just mention here that an ominimal structure on $\mathbb{R}^{n}$ is a collection of subsets of $\mathbb{R}^{n}$ which contains all semi-algebraic subsets, stable under the natural set-theoretic operations and satisfy certain geometric finiteness properties.

In what follows we consider the o-minimal structure $\mathbb{R}_{a n, \exp }$ which is generated by $\mathbb{R}_{a n}$ and $\mathbb{R}_{\text {exp }}$. Here $\mathbb{R}_{a n}$ is the structure afforded by the so-called globally subanalytic sets and $\mathbb{R}_{\text {exp }}$ is the structure consiting of the sets defined by the exponential. In what follows by 'definable' we mean definable in $\mathbb{R}_{\text {an,exp }}$. A function from $A \subset \mathbb{R}^{n}$ to $B \subset \mathbb{R}^{m}$ is said to be definable if its graph in $A \times B \subset \mathbb{R}^{n+m}$ is definable.

We will use recent results of Peterzil-Starchenko (see [9]) which we now describe. We follow section 6.3 of [9]. Let $\mathrm{Sp}_{2 g}$ be the algebraic group (over $\mathbb{Q}$ ) of symplectic $2 g \times 2 g$ matrices with determinant one. The group $\mathrm{Sp}_{2 g}(\mathbb{Z})$ acts on the Siegel upper half space $\mathbb{H}_{g}$. There exists a semi-algebraic subset $\mathcal{F}_{g} \subset \mathbb{H}_{g}$ which contains finitely many representatives for each orbit of $\operatorname{Sp}_{2 g}(\mathbb{Z})$ (hence $\mathcal{F}_{g}$ contains a fundamental domain). For $(a, b) \in \mathbb{R}^{g}$, let $\vartheta_{(a, b)}$ be the corresponding theta function on $\mathbb{H}_{g}$, using the notations of [9]. By definition

$$
\vartheta_{(a, b)}(\tau, z)=\sum_{m \in \mathbb{Z}^{g}} e^{i \pi\left((m-a)^{t} \tau(m+a)+2(m-a)^{t}(z+b)\right)}
$$

for $\tau \in \mathbb{H}_{g}$ and $z$ in the fundamental domain of the lattice in $\mathbb{C}^{g}$ defined by $\tau$.

A special case of the result of Peterzil and Starchenko (theorem 6.5 of [9]), relevant to us, is the following:

Theorem 4.1 (Peterzil-Starchenko) For every $(a, b) \in \mathbb{R}^{g}, \vartheta_{a, b}(z, 0), z \in$ $\mathcal{F}_{g}$ is definable. 
For a subset $\mathcal{Z} \subset \mathbb{R}^{n}$, as in the previous section, the algebraic part of $Z$, denoted by $\mathcal{Z}^{\text {alg }}$, is the union of all connected positive-dimensional semialgebraic subsets of $\mathcal{Z}$. Following Pila, we also denote for $T \geq e$,

$Z(k, T)=\left\{z \in \mathcal{Z}(k):\left[\mathbb{Q}\left(z_{i}\right): \mathbb{Q}\right] \leq k, \max _{i} H\left(z_{i}\right) \leq T\right\}$ and $N_{k}(\mathcal{Z}, T):=|Z(k, T)|$

Theorem 4.2 (Pila-Wilkie) Let $\mathcal{Z} \subset \mathbb{R}^{n}$ be a set definable in an o-minimal structure over $\mathbb{R}$. Let $k \geq 1$ and $\epsilon>0$, there exists $c(\mathcal{Z}, k, \epsilon)$,

$$
N_{k}\left(\mathcal{Z} \backslash \mathcal{Z}^{\text {alg }}, T\right) \leq c(\mathcal{Z}, k, \epsilon) T^{\epsilon}
$$

The consequence of the results of Peterzil-Starchenko and Pila-Wilkie relevant to us is the following:

Theorem 4.3 Let $C$ be an irreducible algebraic curve contained in $S$. Let $\mathcal{F}$ be the set as in section 2. Suppose that $\mathcal{Z}:=\pi^{-1} C \cap \mathcal{F}$ is positive dimensional.

Then for $k \geq 1$ and $\epsilon>0$, there exists $c(\mathcal{Z}, k, \epsilon)$,

$$
N_{k}\left(\mathcal{Z} \backslash \mathcal{Z}^{\text {alg }}, T\right) \leq c(\mathcal{Z}, k, \epsilon) T^{\epsilon}
$$

Proof. Let

$$
S=\Gamma \backslash \mathbb{H}^{2} \longrightarrow \mathrm{Sp}_{2 g}(\mathbb{Z}) \backslash \mathbb{H}_{2}
$$

be the modular embedding (see [16], Chapter IX, $\S 1$ ). This embedding is induced by an equivariant embedding $\phi: \mathbb{H}^{2} \longrightarrow \mathbb{H}_{2}$. After, if necessary replacing the set $\mathcal{F}_{g}$ with a finite union of its images by some $\gamma \in \operatorname{Sp}_{2 g}(\mathbb{Z})$ (this does not affect the conclusion of Peterzil-Starchenko's theorem), we assume that $\phi(\mathcal{F}) \subset \mathcal{F}_{g}$. The set $\mathcal{F}$ is definable (it is semi-algebraic).

The functions $\vartheta_{a, b}(\tau, 0)$ restricted to $\mathbb{H}^{2}$ induce a $\Gamma$-equivariant holomorphic embedding of $S$ into some $\mathbb{P}^{N}(\mathbb{C})$. As $C$ is an algebraic curve, its image in $\mathbb{P}^{N}(\mathbb{C})$ is given by a collection of polynomial equations in the $\vartheta_{(a, b)}(\tau, 0)$ with $\theta \in \mathcal{F}$. It follows from the Paterzil-Starchenko theorem, that the set $\mathcal{Z}$ is definable. The conclusion now follows from the Pila-Wilkie theorem 4.2.

\section{Proof of the main result.}

Let $C$ be a curve in $S$ containing an infinite set $\Sigma$ of special points. Suppose that the closure of $C$ in $\bar{S}$ (Baily-Borel compactification of $S$ ) contains a cusp 
$P$. After, if necessary, replacing $C$ by a component of its image by suitable Hecke correspondence (which does not affect the property of $C$ being special), we assume that $P=\infty$. Let $\mathcal{F}$ be the subset of $\mathbb{H} \times \mathbb{H}$ as in section 2 . Our assumption that $P=\infty$ implies that after possibly replacing $\Sigma$ by an infinite subset, the preimages of the points in $\Sigma$ lie in $\mathcal{F}$. Suppose that $C$ is not special. Let $\mathcal{Z}^{\text {alg }}$ be as in section 3 i.e the algebraic part of $\mathcal{Z}:=\mathcal{F} \cap \pi^{-1} C$. Then $\mathcal{Z}^{\text {alg }}$ contains no special points of $\mathbb{H} \times \mathbb{H}$ by 3.1.

Let $z$ be a point in $\mathcal{F}$ such that $x:=\pi(z) \in \Sigma$. We write $d_{x}$ for the discriminant $d_{z}$ and $A_{x}$ for the isomorphism class of the abelian variety $A_{z}$ as in section 2. Notice that $d_{x}$ and $A_{x}$ depend on $x$ only and not on the choice of a point in $\pi^{-1}(x)$.

As special points are $\overline{\mathbb{Q}}$-valued, $C$ is defined over $\overline{\mathbb{Q}}$ and we can choose a number field $L$ such that $C$ is defined and geometrically irreducible over $L$ Hence for all $x \in \Sigma, \operatorname{Gal}(\overline{\mathbb{Q}} / L) \cdot x$ is contained in $C$.

Let $x$ be a point in $\Sigma$. By theorem 6.2 of [3],

$$
|\operatorname{Gal}(\overline{\mathbb{Q}} / L) \cdot x| \geq c_{2} d_{x}^{1 / 8}
$$

for some absolute constant $c_{2}>0$.

As the points in $\operatorname{Gal}(\overline{\mathbb{Q}} / L) \cdot x$ have the same discriminant $d_{x}$, for any $z \in \mathcal{Z}$ such that $\pi(z) \in \operatorname{Gal}(\overline{\mathbb{Q}} / L) \cdot x$, we have by 2.2

$$
H(z) \leq c_{1} d_{x}^{1 / 4}
$$

On the other hand, by the theorem 4.3, we have for every $\epsilon>0$,

$$
N_{4}\left(\mathcal{Z} \backslash \mathcal{Z}^{\text {alg }}, c_{1} d_{x}^{1 / 4}\right)=N_{4}\left(\mathcal{Z}, c_{1} d_{x}^{1 / 4}\right) \leq c_{\epsilon} c_{1}^{\epsilon} d_{x}^{\epsilon / 4}
$$

for some $c_{\epsilon}$ depending on $\mathcal{Z}$ and $\epsilon$ only. It follows that

$$
c_{\epsilon} c_{1}^{\epsilon} d_{x}^{\epsilon / 4} \geq c_{2} d_{x}^{1 / 8}
$$

i.e

$$
d_{x}^{\frac{1-2 \epsilon}{8}} \leq \frac{c_{\epsilon} c_{1}^{\epsilon}}{c_{2}}
$$

Notice that $d_{x}$ tends to infinity when $x$ ranges through $\Sigma$. Indeed there are only finitely many orders of degree two over $O_{F}$ with a given discriminant and for each of these orders there are finitely many special points $x$ with $\operatorname{End}_{O_{F}}\left(A_{x}\right)$ isomorphic to it.

Choose any $0<\epsilon<\frac{1}{2}$. Then the left hand side of the last inequality goes to infinity as $x$ ranges through $\Sigma$ while the right hand side remains bounded. This yields a contradiction, hence $C$ is a special subvariety. 


\section{Bibliography.}

[1] E. Bombieri, W. Gubler Heights in Diophantine Goemetry. Cambridge University Press, 2006.

[2] D. Cox Primes of the form $x^{2}+n y^{2}$. John Wiley and Sons, Inc. 1989.

[3] B. Edixhoven The André-Oort conjecture for Hilbert modular surfaces. Moduli of abelian varieties (Texel Island, 1999), 133-155, Progr. Math., 195, Birkhuser, Basel, 2001

[4] B. Edixhoven, A. Yafaev Subvarieties of Shimura varieties. Ann. of Math. (2) 157 (2003), no. 2, 621-645.

[5] E. Freitag Hilbert modular forms. Springer, 1990

[6] B. Klingler, A. Yafaev The André-Oort conjecture. Preprint.

[7] B. Moonen Linearity properties of Shimura varieties. I. J. Algebraic Geom. 7 (1998), no. 3, 539-567.

[8] R. Noot Correspondances de Hecke, action de Galois et la conjecture d'Andr-Oort (d'aprs Edixhoven et Yafaev) Séminaire Bourbaki. Vol. 2004/2005. Astérisque No. 307 (2006), Exp. No. 942, vii, 165-197

[9] Y. Peterzil, S. Starchenko Tame complex analysis and o-minimality. Proceedings of the ICM, Hyderabad, 2010. Available on first author's webpage.

[10] J. Pila Algebraic points of definable sets and results of André-OortManin-Mumford type. IMRN, 2009, n. 13, 2476-2507.

[11] J. Pila o-minimality and André-Oort conjecture for $\mathbb{C}^{n}$. Preprint. 2010.

[12] E. Ullmo, A. Yafaev A characterisation of special subvarieties. Preprint. 2010. Availbale on second author's web-page.

[13] E. Ullmo, A. Yafaev Galois orbits and equidistribution: towards the André-Oort conjecture. Preprint.

[14] A. Yafaev The André-Oort conjecture-a survey. L-functions and Galois representations, 381-406, London Math. Soc. Lecture Note Ser., 320, Cambridge Univ. Press, Cambridge, 2007 
[15] A. Yafaev Galois orbits and equidistribution: Manin-Mumford and Andr-Oort J. Thor. Nombres Bordeaux 21 (2009), no. 2, 493-502.

[16] G. Van Der Geer Hilbert modular surfaces. Ergeb. Math. Grenzeb. (3), vol. 16, Springer-Verlag, Berlin, New York, 1987

[17] G. Van Der Geer, K. Ueno Families of abelian surfaces with real multiplications over Hilbert modular surfaces. Nagoya Math. Journal, Vol. 88. 1982, 17-53. 